\title{
Modeling of flexible beam networks and morphing structures by geometrically exact discrete beams
}

\author{
Claire Lestringant \\ Structures Research group, Department of Engineering \\ University of Cambridge, United Kingdom \\ Email: casl4@cam.ac.uk \\ Dennis M. Kochmann* \\ Mechanics \& Materials, Department of Mechanical and Process Engineering \\ ETH Zürich, 8092 Zürich, Switzerland \\ Email: dmk@ethz.ch
}

\begin{abstract}
We demonstrate how a geometrically exact formulation of discrete slender beams can be generalized for the efficient simulation of complex networks of flexible beams by introducing rigid connections through special junction elements. The numerical framework, which is based on discrete differential geometry of framed curves in a time-discrete setting for time- and history-dependent constitutive models, is applicable to elastic and inelastic beams undergoing large rotations with and without natural curvature and actuation. Especially the latter two aspects make our approach a versatile and efficient alternative to higher-dimensional finite element techniques frequently used, e.g., for the simulation of active, shape-morphing, and reconfigurable structures, as demonstrated by a suite of examples.
\end{abstract}

\section{Introduction}

Recent additive manufacturing techniques have incorporated active materials into networks of flexible, slender structural elements, based on, e.g., photo-elastic materials, magnetic actuation, shape memory polymers, and swelling composites [1, 2, 3, 4, 5, 6, 7]. This has provided a new avenue for creating (meta-)materials with engineered properties and time-dependent performance (coined 4D-printing). At the same time, this new opportunity has increased the demand for computational modeling techniques applicable to the aforementioned systems in order to efficiently describe and predict the mechanical response of those advanced structures used for, e.g., multistable, reconfigurable space structures, sensors, soft robots, and flexible electronics [8,9, 10, 11].

Even though these structures are long and slender and thus fall within the scope of one-dimensional (1D) structural theories as shown, e.g., by [4] for thermo-mechanical poly-

\footnotetext{
${ }^{*}$ Address all correspondence to this author.
}

mers, existing modeling work primarily relies on computationally expensive two- and three-dimensional (2D and 3D, respectively) simulations [8,11,12]. Here, we therefore propose a discrete beam framework for modeling networks of flexible, slender elements undergoing large rotations, with special emphasis on versatility in the choice of the underlying material constitutive behavior (including active materials and time-dependent, inelastic constitutive laws such as those found in shape memory polymers) while also providing a computationally efficient alternative to fully-resolved higher-dimensional models.

Geometrically exact discrete beam models were originally introduced in order to numerically solve problems involving slender structures undergoing large rotations [13, 14]. Among the various improvements and extensions that have been proposed since then (see, e.g., [15, 16, 17]), a formulation based on discrete framed curves and discrete parallel transport embeds the unshearability constraint and thus allows rotations to be parametrized by a minimal set of degrees of freedom [18, 19, 20]. This approach has proven its versatility in a variety of applications ranging from elastic beams [18] to inextensible elastic ribbons [21] to viscous threads [22, 23] to viscoelastic rods [24, 25] as well as to problems including contact, self-contact and friction [26, 27, 28].

By contrast to previous formulations that were specific to a particular type of constitutive behavior [18, 21, 22, 23 . 24, 25], we recently proposed [29] a geometrically exact discrete beam element formulation adapted from [18], in which the beam kinematics is separated from the constitutive law of the underlying base material, hence making the modular descriptions applicable to various elastic and inelastic constitutive laws, in a time-discrete framework based on variational consitutive updates [30]. Unlike, e.g., corotational beam formulations [31, 32], our approach describes beam 
bending without the introduction of rotational degrees of freedom but through the kinematic extraction of an effective beam curvature from the positions of the vertices of a discrete framed curve. It is for this reason (and the low-order interpolation introduced by this discretization) that it has so far been impossible to control rotations at vertices along the discrete curve, which prevents the modeling of rigid connections between segments in a network or complex boundary conditions such as clamped beams. Therefore, [29] focused on modeling individual beams (i.e., having the topology of a segment). Here, we show that this limitation can be circumvented by redistributing the degrees of freedom at the level of one element and introducing rigid-body rotations. This greatly extends the existing model towards taking into account rigid junctions between beams (e.g., in order to model welded joints in beam lattices and truss metamaterials, as shown in the numerical examples).

In order to extend the formulation and to overcome the present limitations, one can introduce a coupling energy depending on the the rigid rotation at a junction. The solution proposed by [33] involves first finding a linear transformation at every junction and then extracting the associated finite rotation by polar decomposition - which can come with significant computational costs. Here, by contrast, we introduce a new technique based on a virtual (ghost) segment on which a rigid rotational constraint is imposed, thus avoiding least-square fitting and the use of a polar decomposition. Moreover, contrary to [33] we do not limit ourselves to elastic rods but intend to make the framework applicable to a wide catalog of constitutive laws (including time- and history-dependent behavior), following the same approach based on variational constitutive updates presented in [29]. As an added benefit, our junction elements can be connected to other types of finite element discretizations involving rotational degrees of freedom at vertices, such as the classical corotational beam elements of [31].

In the following Section 2, we briefly summarize the theoretical-numerical framework for modeling geometrically exact discrete nonlinear, flexible beams with special focus on the treatment of rotations and the extension to networks of slender beams through the introduction of rigid junction elements. Section 3 is dedicated to model validation in terms of a quantitative comparison of our new framework with a classical corotational beam formulation. To demonstrate the ability of our code to predict the behavior of shape-morphing networks, Section 4 presents selected examples of actuated beam networks and 4D-printed structures (drawing inspiration from recent work on active metamaterials and reconfigurable structures), before Section 5 concludes this study.

\section{Theoretical-numerical framework for discrete beam networks}

Based on the discrete beam model introduced in [29], our formulation combines a kinematic description for discrete framed curves undergoing large rotations [18] with variational constitutive updates [30] in a holistic framework. We use the notion of parallel transport in time to parametrize

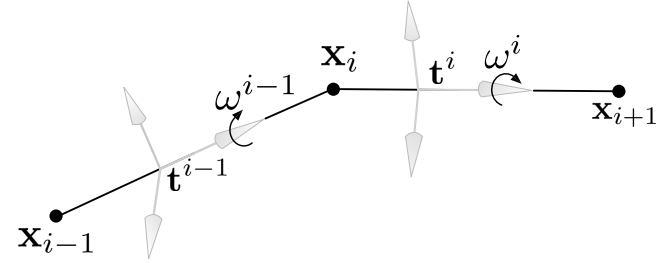

Fig. 1: Discrete beam element of index $i$. Portion of the discrete line parametrized by the vector of local degrees of freedom $\left(\mathbf{x}_{i-1}, \mathbf{x}_{i}, \mathbf{x}_{i+1}, \omega^{i-1}, \omega^{i}\right)$.

rotations locally on every segment of the discrete curve, while adopting an updated-Lagrangian setting suitable for large rotations. We start with a brief summary of the approach introduced in [29] for modeling geometrically exact slender beams, only laying out those key concepts required here for subsequent derivations and discussions. Within this framework, we particularly demonstrate how to control rotations at vertices.

\subsection{Parametrization of discrete beams}

In our time-discrete updated-Lagrangian approach, the reference configuration denotes the beam configuration at the previous time step, while the current configuration refers to the beam in its current state (i.e., at the end of the latest time step). Identifying by an asterisk $\left(_{\star}\right)$ all quantities related to the reference configuration (while all quantities carrying no asterisk are defined in the current configuration), we describe the centerline of a beam by a discrete line spanned by $n$ vertices at positions $\left\{\left(\mathbf{x}_{0}\right)_{\star}, \cdots,\left(\mathbf{x}_{n-1}\right)_{\star}\right\}$ in the reference configuration and $\left\{\mathbf{x}_{0}, \cdots, \mathbf{x}_{n-1}\right\}$ in the current configuration. Here and in the following, whenever a quantity is defined analogously in the reference and current configuration, we simply present one definition (and the respective other one follows analogously by addition/removal of the asterisk).

Each pair of adjacent vertices $\left(\mathbf{x}_{k}, \mathbf{x}_{k+1}\right)$ for $k \in\{0, n-$ 2) spans a segment of index $k$. For each segment we define a unit tangent $\mathbf{t}^{k}=\mathbf{e}^{k} /\left\|\mathbf{e}^{k}\right\|$ as the normed vertex-to-vertex vector $\mathbf{e}^{k}=\mathbf{x}_{k+1}-\mathbf{x}_{k}$, see Fig. 1. To avoid confusion, we label all segment-based quantities with superscripts, while vertex-based quantities are labeled with subscripts. The orientation of segment $k$ 's cross-section is captured by the material frame $\left(\mathbf{d}_{1}^{k}, \mathbf{d}_{2}^{k}, \mathbf{d}_{3}^{k}\right)$, which defines an orthonormal triad aligned such that $\mathbf{d}_{3}^{k}=\mathbf{t}^{k}$. This latter geometric constraint ensures the unshearability of the discrete beam, although an extension to shearable rods is available without major difficulty [28]. As shown in [29], the change of orientation between the reference and current configurations is expressed as a composition of rotations, viz.

$$
\mathbf{d}_{I}^{k}=\mathbf{P}_{\mathbf{t}_{\star}^{k}}^{\mathbf{t}^{k}} \cdot \mathbf{R}\left(\mathbf{t}_{\star}^{k}, \omega^{k}\right) \cdot\left(\mathbf{d}_{I}^{k}\right)_{\star} \quad \text { for } \quad I \in\{1,2,3\},
$$

where $\mathbf{R}(\mathbf{u}, \beta) \in \mathrm{SO}(3)$ denotes the rotation by an angle $\beta$ about unit vector $\mathbf{u}$, and the angle $\omega^{k}$ is identified as the integral of the spinning velocity (i.e., the torsional component of the segment's angular velocity) over the time step. (1) uses 
the parallel transport $\mathbf{P}_{\mathbf{t}_{\star}^{k}}^{\mathbf{t}^{k}}$, which denotes a specific rotation defined as follows. For two unit vectors $\mathbf{v}_{1}, \mathbf{v}_{2} \in \mathbb{R}^{3}, \mathbf{P}_{\mathbf{v}_{1}}^{\mathbf{v}_{2}}$ denotes the rotation about the vector $\mathbf{v}_{1} \times \mathbf{v}_{2}$ which maps $\mathbf{v}_{1}$ onto $\mathbf{v}_{2}$. When the two vectors $\mathbf{v}_{1}$ and $\mathbf{v}_{2}$ are equal, $\mathbf{P}_{\mathbf{v}_{1}}^{\mathbf{v}_{2}}$ is simply the identity. Properties, explicit expressions as well as a practical way to evaluate the parallel transport relations were provided in [29] and are omitted here for conciseness.

With the above choice of discretization, the current configuration is parametrized by the global vector of degrees of freedom,

$$
\mathbf{u}=\left(\mathbf{x}_{0}, \omega^{0}, \cdots, \mathbf{x}_{i-1}, \omega^{i-1}, \mathbf{x}_{i}, \omega^{i}, \mathbf{x}_{i+1}, \cdots, \omega^{n-2}, \mathbf{x}_{n-1}\right)
$$

containing all vertex positions $\mathbf{x}_{k}(k=1, \ldots, n-1)$ as well as all segment spin angles $\omega^{i}(i=0, \ldots, n-2)$.

We define the discrete beam element of index $i$ ( $i \in$ $\{1, \cdots, n-2\})$ as the portion of a discrete line spanned by a triad of nodes (centered at vertex $i$ ) at positions $\left\{\mathbf{x}_{i-1}, \mathbf{x}_{i}, \mathbf{x}_{i+1}\right\}$ in the current configuration, as illustrated in Figure 1. With our choice of interpolation, this is the smallest portion capturing twisting and bending strains, as shown below. The local degrees of freedom associated with the $i$ th element (as shown in Figure 1) are extracted from the global vector of degrees of freedom (2) by a connectivity matrix $\mathbf{C}_{i}$ (whose coefficients are either 0 or 1 ) according to

$$
\mathbf{u}_{i}=\mathbf{C}_{i} \cdot \mathbf{u}=\left(\mathbf{x}_{i-1}, \mathbf{x}_{i}, \mathbf{x}_{i+1}, \omega^{i-1}, \omega^{i}\right)
$$

\subsection{Controlling rotations at vertices}

With the degrees of freedom of the discrete beam element defined by (3), rotations unfortunately cannot be controlled explicitly at vertices; this excludes the application of clamped boundary conditions as well as rigid connections within beam networks. Here, we overcome this limitation by introducing a new junction element comprising two adjacent vertices and tied to a rigid-body rotation, as sketched in Figure 2(a). As illustrated in the example, the shown element $i$ contains only a single (physical) segment (defined by the two vertices $i-1$ and $i$ at positions $\left\{\mathbf{x}_{i-1}, \mathbf{x}_{i}\right\}$ ), while the second segment is replaced by a virtual ghost segment implementing the rigid-body rotational constraint. Specifically, vertex $i$ is tied to a rigid-body rotation parametrized by a unit quaternion labeled $\mathbf{q}^{i} \in \mathbb{R}^{4}$ or, alternatively, by the corresponding rotation tensor $\mathbf{R}^{i} \in \mathrm{SO}(3)$ (see appendix A for details about the parametrization of rotations and the relation between $\mathbf{q}^{i}$ and $\mathbf{R}^{i}$ ).

The local degrees of freedom associated with this element are defined as

$$
\mathbf{u}_{i}=\left(\mathbf{x}_{i-1}, \mathbf{x}_{i}, \omega^{i-1}, \mathbf{q}^{i}\right)
$$

The orientation of the ghost segment at vertex $i$ is controlled by the rigid rotation parametrized by $\mathbf{q}^{i}$ (the superscript $i$ indicating that the rotation is in fact a quantity pertaining to a segment). This ghost segment is characterized by a unit tangent $\tilde{\mathbf{t}}^{i}$, a spin angle $\tilde{\omega}^{i}$ and a material frame $\left(\tilde{\mathbf{d}}_{1}^{i}, \tilde{\mathbf{d}}_{2}^{i}, \tilde{\mathbf{d}}_{3}^{i}\right)$. Note that we use an updated Lagrangian approach: the degrees of freedom $\mathbf{q}^{i}$ therefore define an incremental rotation (that parametrizes the current configuration with respect to the reference configuration). As a result, the tangent vector $\tilde{\mathbf{t}}^{i}$ in the current configuration is obtained by applying the rotation to $\tilde{\mathbf{t}}_{\star}^{i}$ in the reference configuration:

$$
\tilde{\mathbf{t}}^{i}=\mathbf{R}^{i} \cdot \tilde{\mathbf{t}}_{\star}^{i}
$$

Furthermore, we observe that the composition of the rotation $\mathbf{R}^{i}$ (applied first) with the parallel transport $\mathbf{P}_{\tilde{\mathbf{t}}^{i}}^{{\tilde{\mathfrak{t}^{i}}}^{i}}$ that brings back $\tilde{\mathbf{t}}^{i}$ to $\tilde{\mathbf{t}}_{\star}^{i}$ (applied second) leaves $\tilde{\mathbf{t}}_{\star}^{i}$ invariant and can be identified as the rotation of angle $\tilde{\omega}^{i}$ about $\tilde{\mathbf{t}}_{\star}^{i}$, so that the spin angle $\tilde{\omega}^{i}$ is given implicitly by the relation

$$
\mathbf{R}\left(\tilde{\mathbf{t}}_{\star}^{i}, \tilde{\omega}^{i}\right)=\mathbf{P}_{\tilde{\mathbf{t}}^{i}}^{\tilde{\mathbf{t}}^{i}} \cdot \mathbf{R}^{i}
$$

Practically, vertex $i$ serves as the endpoint of a beam, to which one may wish to impose a boundary condition (such as an external moment, a clamping condition or a rigid connection to another beam). For the example in Figure 2(a), a clamping boundary condition perpendicular to the $\left(\mathbf{e}_{x}, \mathbf{e}_{z}\right)$ plane (with $\left\{\mathbf{e}_{x}, \mathbf{e}_{y}, \mathbf{e}_{z}\right\}$ denoting the Cartesian basis) is applied at vertex $i$ by choosing $\tilde{\mathbf{t}}_{\star}^{i}=\mathbf{e}_{y},\left(\tilde{\mathbf{d}}_{1}^{i}\right)_{\star}=\mathbf{e}_{z}$ and $\left(\tilde{\mathbf{d}}_{2}^{i}\right)_{\star}=$ $\mathbf{e}_{x}$ at the beginning of the calculation and by imposing $\mathbf{R}^{i}$ to be the identity, i.e. $\mathbf{q}^{i}=(1,0,0,0)$, as an essential boundary conditions at every time step.

Note that the relations between the local vector of degrees of freedom (4), the tangent vector $\tilde{\mathbf{t}}^{i}$, and the spin vector $\tilde{\omega}^{i}$ of the ghost segment are nonlinear (especially, $\tilde{\omega}^{i}$ depends nonlinearly on $\mathbf{q}^{i}$ through the implicit equation (6). This complicates the calculation of the conjugate forces and of the consistent tangent matrix of the junction element introduced here, as summarized in appendix B For small time steps and hence small increments in rotation, the current configuration is close to the reference configuration, so $\mathbf{R}^{i} \approx \mathbf{I}$ and $\tilde{\mathbf{t}}^{i} \cdot \tilde{\mathbf{t}}_{\star}^{i} \approx 1$. In this case the rotation $\mathbf{R}\left(\tilde{\mathbf{t}}_{\star}^{i}, \tilde{\boldsymbol{\omega}}^{i}\right)$ can be approximated by an infinitesimal rotation about $\tilde{\mathbf{t}}_{\star}^{i}$ writes explicitly

$$
\mathbf{R}\left(\tilde{\mathbf{t}}_{\star}^{i}, \tilde{\omega}^{i}\right) \approx \mathbf{I}+\tilde{\omega}^{i}\left(\tilde{\mathbf{t}}_{\star}^{i}\right)_{\times},
$$

where, for any vector $\mathbf{v}, \mathbf{v}_{\times}$denotes a skew-symmetric tensor such that $\mathbf{v}_{\times} \cdot \mathbf{x}=\mathbf{v} \times \mathbf{x}$ for all $\mathbf{x} \in \mathbb{R}^{3}$. Combined with (6), this yields $\mathbf{I}+\tilde{\omega}^{i}\left(\tilde{\mathbf{t}}_{\star}^{i}\right)_{\times} \approx \mathbf{P}_{\tilde{\mathbf{t}}^{i}}^{\tilde{\tilde{t}}^{i}} \cdot \mathbf{R}^{i}$, and it follows that

$$
\tilde{\omega}^{i} \approx \frac{1}{2}\left[\mathbf{P}_{\tilde{\mathbf{t}}^{i}}^{\tilde{\tilde{t}}^{i}} \cdot \mathbf{R}^{i}\right]:\left(\tilde{\mathbf{t}}_{\star}^{i}\right)_{\times}
$$

The general case of $\mathbf{R}^{i}$ being a finite rotation is detailed in appendix $\mathrm{B}$. Once $\tilde{\mathbf{t}}^{i}$ and $\tilde{\omega}^{i}$ are known, the material frame vectors $\left(\widetilde{\mathbf{d}}{ }_{1}^{i}, \tilde{\mathbf{d}}_{2}^{i}, \tilde{\mathbf{d}}_{3}^{i}\right)$ in the current configuration follow from applying (1). 


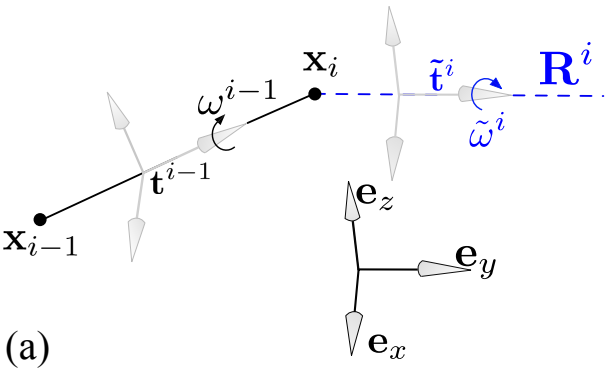

(a)

Fig. 2: Rigid junctions between discrete beams. (a) A discrete beam element tied to a rigid-body rotation is realized by a junction element consisting of only a single physical segment tied to a virtual segment parametrized by a rigid rotation $\mathbf{R}^{i}$. The junction element hence has degrees of freedom $\left(\mathbf{x}_{i-1}, \mathbf{x}_{i}, \omega^{i-1}, \mathbf{q}^{i}\right)$ where $\mathbf{q}^{i}$ denotes the quaternion of the rigid rotation. The angular spin $\tilde{\omega}^{i}$ and the unit tangent $\tilde{\mathbf{t}}^{i}$ of the virtual segment are reconstructed from the rotation $\mathbf{R}^{i}$ through (5) and (6). (b) Two discrete beam elements (represented in blue and red) are rigidly connected at node $k$, whose rotation is parametrized by $\mathbf{R}^{k}\left(\mathbf{q}^{k}\right)$. The local degrees of freedom of the two elements are respectively $\left(\mathbf{x}_{k-1}, \mathbf{x}_{k}, \omega^{k-1}, \mathbf{q}^{k}\right)$ (element in blue), and $\left(\mathbf{x}_{k+1}, \mathbf{x}_{k}, \omega^{k}, \mathbf{q}^{k}\right)$ (element in red).

With the above junction element, a discrete beam can be tied at one of its end vertices to a rigid-body rotation by redistributing its degrees of freedom: one of the two vertices of a junction element (vertex $i$ in Figure2 2 a)) becomes the end vertex of the beam, which effectively ties the last physical beam segment (between vertices $i-1$ and $i$ in Figure 2(a)) to a rigid-body rotation through the ghost segment. Rigid junctions between several discrete beams are consequently implemented by combining several junction elements, as illustrated in Figure 2(b). In addition, it is possible to connect a discrete beam element to a beam element based on a parametrization involving rotational degrees of freedom at vertices (such as the classical corotational beam elements of [31]).

We point out that the introduction of the ghost segment increases the book-keeping complexity associated with the junction elements, since the (global) ordering of vertices must differentiate between the physical and virtual segments (they carry different degrees of freedom and are treated differently). The local ordering of degrees freedom within a junction element falls in one of the two cases depicted in Figure 2 b), depending on whether the ghost segment is connected to the first or to the second vertex of the junction element. We close by noting that one can alternatively parametrize rotations using pseudovectors instead of quaternions without changing the overall structure of the above formulation.

\subsection{Discrete beam strains}

With elastic, vicous, and viscoelastic constitutive models in mind for subsequent applications, we choose to extract a set of strain measures from the above beam kinematics, including axial, flexural, and torsional strains. To this end, we define for the $i$ th beam element the vector of strains

$$
\mathbf{E}_{i}=\left(\varepsilon_{i}, \kappa_{i}^{1}, \kappa_{i}^{2}, \tau_{i}\right)
$$

where $\varepsilon_{i}$ denotes the discrete axial strain, $\kappa_{i}^{1}$ and $\kappa_{i}^{2}$ are the bending strains about the two axes defined by the material frame, and $\tau_{i}$ denotes the torsional/twisting strain.

Specifically, for beam elements, we define the discrete axial strain as

$$
\varepsilon_{i}=\frac{1}{2}\left(\frac{\left\|\mathbf{e}^{i-1}\right\|-l_{0}^{i-1}}{l_{0}^{i-1}}+\frac{\left\|\mathbf{e}^{i}\right\|-l_{0}^{i}}{l_{0}^{i}}\right)
$$

where $l_{0}^{i}$ and $l_{0}^{i-1}$ denote the initial, undeformed lengths of the segments spanned by, respectively, $\mathbf{e}^{i}$ and $\mathbf{e}^{i-1}$. We further define the bending strains as

$$
\kappa_{i}^{I}=\mathbf{k}_{i} \cdot \frac{\mathbf{d}_{I}^{i-1}+\mathbf{d}_{I}^{i}}{2} \quad \text { for } \quad I \in\{1,2\}
$$

with the vertex-based discrete curvature binormal $\mathbf{k}_{i}=$ $2 \mathbf{t}^{i-1} \times \mathbf{t}^{i} /\left(1+\mathbf{t}^{i-1} \cdot \mathbf{t}^{i}\right)$. Finally, the discrete twisting strain in the current configuration can be expressed as

$$
\tau_{i}=\left(\tau_{i}\right)_{\star}+\omega^{i}-\omega^{i-1}+\gamma_{i}
$$

where $\gamma_{i}$ can be calculated as the spherical area of the polygon spanned by vectors $\left(\mathbf{t}_{\star}^{i-1}, \mathbf{t}_{\star}^{i}, \mathbf{t}^{i}, \mathbf{t}^{i-1}\right)$. Detailed derivations of this expression for the twist (as well as all other kinematics) can be found in [29]. Note that the bending and twisting strain measures defined above are integrated quantities, as discussed in [29] and in the presentation of constitutive laws below.

In the case of a junction element, $\varepsilon_{i}$ simply represents the axial strain in its physical segment (so that no averaging as in (10) is required), while the virtual quantities (unit tangent, material frame vectors, and spin angle) controlled by the rotation $\mathbf{q}^{i}$ enter the bending and torsional strains through 11 
and (12) as for a regular beam element. As an example, for the element drawn in Figure 2(a) the virtual tangent $\tilde{\mathbf{t}}^{i}$ (or $\tilde{\mathbf{t}}_{\star}^{i}$ ) is used instead of $\mathbf{t}^{i}$ (or $\mathbf{t}_{\star}^{i}$ ), $\omega^{l}$ is replaced by $\tilde{\omega}^{i}$, and the virtual material frame vectors $\tilde{\mathbf{d}}_{1}^{i}\left(\right.$ or $\left.\left(\tilde{\mathbf{d}}_{1}^{i}\right)_{\star}\right)$ and $\tilde{\mathbf{d}}_{2}^{i}\left(\right.$ or $\left.\left(\tilde{\mathbf{d}}_{2}^{i}\right)_{\star}\right)$ are used instead of $\mathbf{d}_{1}^{i}\left(\right.$ or $\left.\left(\mathbf{d}_{1}^{i}\right)_{\star}\right)$ and $\mathbf{d}_{2}^{i}\left(\right.$ or $\left.\left(\mathbf{d}_{2}^{i}\right)_{\star}\right)$.

\subsection{Constitutive laws}

The above beam and junction elements only define the kinematics of the beam element, whereas the constitutive behavior is introduced independently through a strain energy density (and a dissipation potential in case of time- and/or history-dependent material behavior) in a time-discrete, variational manner. Based on the strain measures (9), we define those potentials depending on the axial, flexural, and torsional beam strains. Here, we focus on the representative cases of linear elastic and viscoelastic constitutive laws, while the approach is sufficiently general to extend to other material behavior.

For a beam made of a homogeneous, isotropic, linear elastic material with Young's modulus $E$ and shear modulus $\mu$, we define the elastic energy $W_{i}$ of the $i$ th beam element as a function of the vector of the strain measures, which writes

$W_{i}\left(\mathbf{E}_{i}\right)=\frac{\tilde{l}_{0}^{i}}{2}\left[E\left(A \varepsilon_{i}^{2}+I_{1}\left(\frac{\kappa_{i}^{1}}{\tilde{l}_{0}^{i}}\right)^{2}+I_{2}\left(\frac{\kappa_{i}^{2}}{\tilde{l}_{0}^{i}}\right)^{2}\right)+\mu J\left(\frac{\tau_{i}}{\tilde{l}_{0}^{i}}\right)^{2}\right]$

where $A$ is the cross-sectional area, $I_{1}$ and $I_{2}$ are the area moments of inertia (with respect to the undeformed, initial material frame axes), and $J$ is the torsion constant. We note that the linear elastic constitutive law is applicable if strains remain small (in particular $\left|\varepsilon_{i}\right| \ll 1$ ), so that any dependence of $A, I_{1}, I_{2}$ and $J$ on deformation is neglected here (for a more general formualtion see [29]). Further note that (13) can easily be adapted to account for eigenstrains (generated, e.g., by thermal actuation), as will be illustrated in Section 4. In order to capture the strain energy due to bending and torsion, we have introduced the undeformed Voronoi length $\tilde{l}_{0}^{i}$ of beam element $i$ as the average $\left(l_{0}^{i-1}+l_{0}^{i}\right) / 2$ of both undeformed, initial segment lengths, whereas for the junction element the undeformed Voronoi length simply represents the undeformed, initial length of its physical segment. Note that the bending and twisting strain measures rescaled by the Voronoi length (i.e., $\kappa_{i}^{I} / \tilde{l}_{0}^{i}$ for $I \in\{1,2\}$ and $\tau_{i} / \tilde{l}_{0}^{i}$ ) converge to the continuous measures of strain in the limit of a zero segment length, as discussed in [29]. For this reason, the strain energy [13] is a function of those rescaled strains.

As an inelastic alternative, we consider slender inextensible beams made of a homogeneous, isotropic standard linear viscoelastic solid with elastic moduli $E_{1}, E_{2}$ and viscosity $\eta$. The constitutive law is modeled by defining a strain energy and a dissipation potential per beam element (confining the description to 2D for simplicity) as, respectively,

$$
\begin{aligned}
W_{i}\left(\mathbf{E}_{i}, z_{i}\right) & =\frac{E_{1} A \tilde{l}_{0}^{i}}{2} \varepsilon_{i}^{2}+\frac{I_{1} \tilde{l}_{0}^{i}}{2}\left[E_{1}\left(\frac{\kappa_{i}^{1}}{\tilde{l}_{0}^{i}}\right)^{2}+E_{2}\left(\frac{\kappa_{i}^{1}-z_{i}}{\tilde{l}_{0}^{i}}\right)^{2}\right], \\
\mathcal{D}_{i}\left(\dot{z}_{i}\right) & =\frac{\eta I_{1}}{2 \tilde{l}_{0}^{i}}\left(\frac{\dot{z}_{i}}{\tilde{l}_{0}^{i}}\right)^{2} .
\end{aligned}
$$

Note that we do not consider viscous effects associated with axial strains, as beams are considered inextensible ( $A L^{2} / I_{1} \gg 1$, where $L$ is the beam's length) in our examples (and there is no torsional strain in 2D). Internal variable $z_{i}$ represents the inelastic change of the reference (integrated) curvature of element $i$. The above potentials are used in a time-incremental fashion, following the formulation of variational constitutive updates [30]. Of course, the same potentials can be defined analogously in $3 \mathrm{D}$, which is omitted here for conciseness.

The complete governing equations for discrete rods are thus obtained by combining the discrete nonlinear strain measures (10), (11), and 12) with the above discrete constitutive potentials; a detailed derivation for discrete beams without junctions was presented in [29].

\subsection{Solving (initial) boundary value problems}

In the following, we solve quasistatic and dynamic (initial) boundary value problems in $2 \mathrm{D}$ and $3 \mathrm{D}$, whose systems of nonlinear governing equations is obtained by combining the discrete energy and/or dissipation potentials described in 'Section 2.4 with the definition of the discrete strains defined in 2.3 in a time-discrete variational setting. The resulting quasistatic force balance equations are solved by NewtonRaphson iteration, while dynamic problems are solved by an implicit time stepping scheme. For segments pertaining to junction elements, inertia is modeled by introducing discrete consistent and lumped mass matrices, following the approach of [29], with no inertia associated with the rotational degrees of freedom of all ghost segments.

Our discrete beam model is implemented in a $\mathrm{C}++$ library, which separates the beam kinematics from the base material's constitutive description for a versatile tool-set that adopts the variational structure of our discrete beam formulation. The modular nature of the code allows for a straightforward addition of new constitutive laws, requiring minimum coding effort by the user. The discrete beam model yields sparse (or banded, when only one beam is involved) stiffness matrices and its implementation has been parallelized by recourse to the PETSc library [34], which admits simulations of large beam networks. The code - used for all examples reported here - is available at github.com/lclaire/UtoBeams.

\section{Validation examples}

In order to validate the above discrete beam formulation along with the new capability of handling beam junctions (and hence truss and frame networks), we first present simple but instructive examples, before preceding to more 


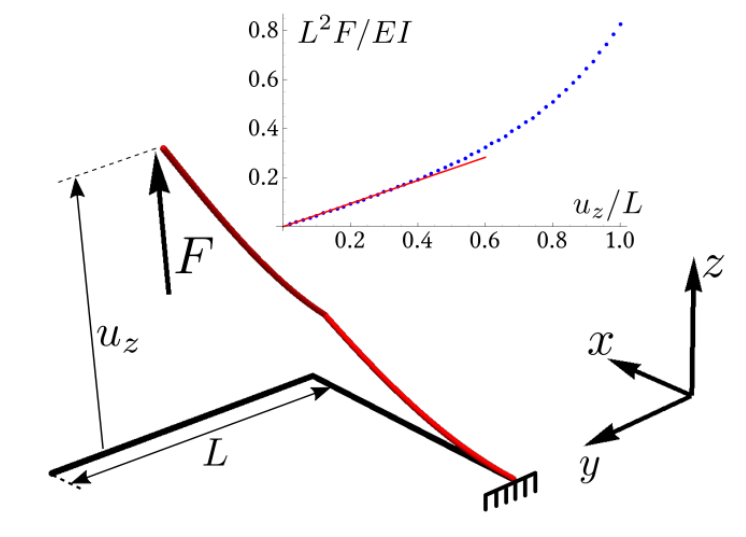

(a)

(b)

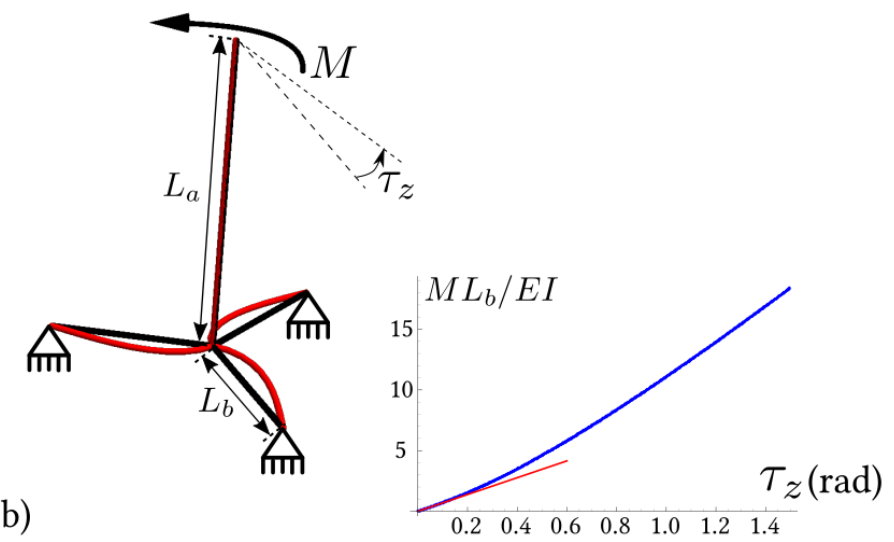

Fig. 3: Validation examples involving a single beam junction (undeformed configurations are shown in black, deformed configurations in red): (a) clamped right-angled frame subjected to an out-of-plane tip displacement, (b) twisting of a windmill structure loaded at the end of its vertical axis and attached at the end of each of its blades. The insets illustrate the (normalized) force-displacement and torque-twist angle curves (in blue) in (a) and (b), respectively, together with the analytical solutions for small deformation (in red).

complex boundary value problems to showcase the versatility of the presented techniques and the impact of eigenstrains. We select two test cases featuring rigid connections, both shown in Figure 3 . (a) an out-of-plane bending test of a two-beam frame welded under a right angle (both beams are of side length $L$ ), which is clamped at one end and subjected to an out-of-plane displacement $u_{z}$ at its other endpoint; (b) a windmill-shaped network of four rigidly welded beams, comprising three in-plane horizontal blades attached at their ends (each of length $L_{b}=L_{a} / 2$ ) which are connected to a vertical beam (length $L_{a}$ ). The structure is subjected to a twist angle $\tau_{z}$ applied at the top end of the vertical beam.

We consider linear elastic beams described by the potential (13) with $\mu=E / 3$ (effectively enforcing incompressibility) having symmetric cross-sections so that $I_{1}=I_{2}=I$. For the frame of Figure $3 \mathrm{a}$, we further take $J=2 I$ and $A L^{2} / I=4.10^{2}$. The torsional rigidity of the mill's vertical axis in Figure $3 \mathrm{p}$ is chosen to be larger than its bending rigidity by taking $J=200 I$ and $A L_{a}^{2} / I=4.10^{2}$, whereas the blades are characterized by $J=I$ and $A L_{b}^{2} / I=10^{2}$. With this set of parameters and in the limit of small displacements, the relation between the pulling force $F$ and the (normalized) vertical displacement of at the frame's tip is found as $\frac{u_{z}}{L}=\frac{14}{6} \frac{F L^{2}}{E I}$. The linear relation between the moment $M$ and twist $\tau_{z}$ at the mill's tip is $\tau_{z}=\frac{13}{90} \frac{M L_{b}}{E I}$ in the limit of small twist and bending. For large displacements, unfortunately, no analytical solutions are available for these benchmarks, and results shown in insets of Figure 3 show significant nonlinearity (and agreement in the limit of small deformation). For validation and a convergence study, we compare our results to simulation results based on the well-established corotational beam element of [31] for comparison [35]. (In the latter formulation, rigid connections between beams, and analogously clamped boundary conditions, are naturally en- forced through (shared) rotational degrees of freedom explicitly defined at vertices for all elements.) We compute the magnitude of the force $F$ (in test (a)) and moment $M$ (in test (b)) which are measured at, respectively, an applied vertical displacement $u_{z} / L=1$ in (a) and a twist angle $\tau_{z}=1.5$ of the vertical shaft in (b). (Details about the calculation of the moment conjugate to an imposed rotation are given in appendix C) Comparison runs using the corotational discrete beam element for approximately (a) 400 and (b) 1900 degrees of freedom. The relative error of our implementation is calculated as

$$
\delta F=\frac{\left|F-F_{\text {corotational }}\right|}{F_{\text {corotational }}}, \quad \delta M=\frac{\left|M-M_{\text {corotational }}\right|}{M_{\text {corotational }}} .
$$

Figure 4 demonstrates the convergence of our results towards the corotational solution with an increasing number of degrees of freedom, $n$. Nearly linear convergence is observed for the two cases (with a slightly sub-linear scaling in case (b)). Note that this is due to our particular choice for the error estimate [15]; in [29] quadratic convergence was observed for problems involving combined bending and torsion using a discrete version of the $L^{2}$-norm. We do not use the discrete $L^{2}$-norm in this study because of the difference in the order of interpolation between our formulation and the corotational beam elements.

A fair comparison of computational costs with existing schemes is challenging due to differences in code architecture and performance scaling as well as solver algorithms (besides any such efficiency metric being specific to a boundary value problem). Here and in the following, we therefore refrain from presenting cost metrics and instead report the number of degrees of freedom used in simulations, as the computational costs scale to first order linearly with the 

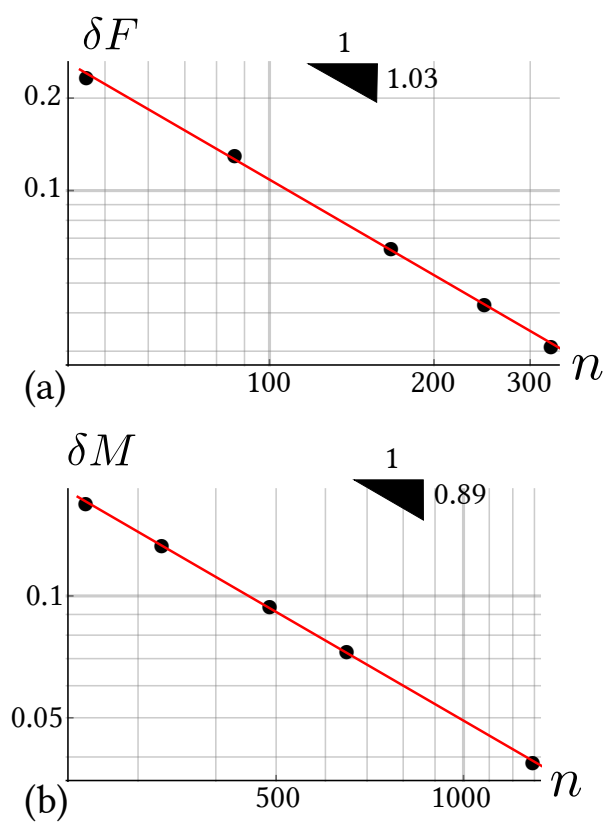

Fig. 4: Convergence of the force/torque conjugate to the applied displacement/twist angle as defined in Figure 3 Results are obtained from the discrete beam implementation with $n$ degrees of freedom, errors are computed against the analogous results predicted with the corotational beam model of [31] with about (a) 400 and (b) 1900 degrees of freedom.

number of degrees of freedom. Figure 4, e.g., demonstrates how accuracy is gained with decreasing efficiency (errors are computed relative to high-fidelity corotational beam simulations with (a) 400 and (b) 1900 degrees of freedom).

\section{Shape-morphing and inelastic networks of slender beams and ribbons}

Numerous shape-changing mechanisms have been exploited in recent years for the design of active and reconfigurable structures, including single-member actuation [36, 37], selective or cooperative buckling [38, 39, 40, 41, 12] and activation of natural curvature or axial eigenstrains [42, 43. 44, 4. 12]. In practice, such mechanisms can be actuated by purely mechanical devices [39, 40, 41] as well as by multiphysics coupling such as, e.g., thermo-mechanical effects, photo-elasticity, and electro-mechanical coupling [42, 43,44 4, 12]. Experimental advances call for simulation techniques that describe and predict such complex structural responses, and we here demonstrate the suitability of our computational framework to investigate different actuation mechanisms in shape-changing networks of slender beams. Specifically, we investigate (i) the time evolution of a viscoelastic 2D network, (ii) the activation of natural curvature in shapechanging elastomeric networks, (iii) the cooperative buckling of a square beam lattice subjected to axial extension due to swelling, and (iv) the self-assembly of 3D ribbon structures by compressive buckling.

\subsection{Viscoelastic membrane}

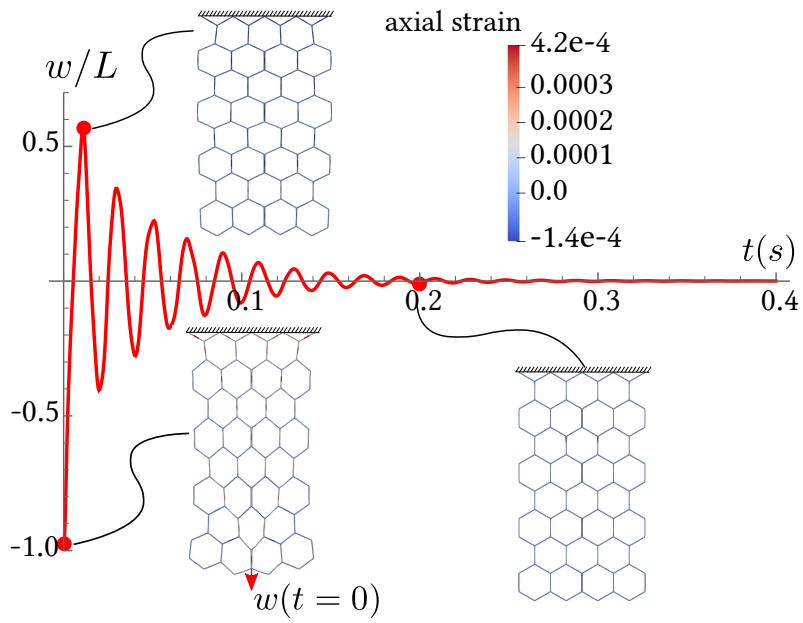

Fig. 5: Time evolution of a viscoelastic hexagonal planar truss clamped along its upper boundary and initially pulled down, calculated with three segments per beam. The dimensionless vertical displacement of the midpoint of the lower boundary is plotted as a function of time. Insets show the deformed configurations at the three times $t \in\{0 \mathrm{~s}, 0.01 \mathrm{~s}, 0.2 \mathrm{~s}\}$; the value of the axial strains within the discrete beam elements is shown by the color map (note that all strains remain small).

We begin by simulating the time evolution of a network of slender beams with a time-dependent material behavior. We study the dynamics of a planar hexagonal truss (consisting of identical, rigidly welded beams of lengths $L$ ) clamped along its upper boundary and initially pulled down by applying a vertical displacement at the midpoint of its lower boundary, as shown in the insets in Figure 5 The beams are made of a standard viscoelastic material approximated by the potentials 114 with a relaxation time $t_{r}=\eta / E_{2} \approx 0.00334 \mathrm{~s}$ and a contrast in moduli $E_{2}=0.3 E_{1}$. We consider slender, quasi-inextensible beams with $I_{1}=I_{2}=I$ and $A L^{2} / I=10^{2}$ and the density $\rho$ is chosen such that the typical time scale of elastic bending oscillations for a single beam of length $L$ amounts to $t_{e}=\sqrt{\rho A /\left(E_{1} I L^{-4}\right)} \approx 0.00139 \mathrm{~s}$, so that the Deborah number (for single beam oscillations) is approximately $\mathrm{De}=t_{r} / t_{e} \approx 2.4$.

The time evolution of the vertical displacement $w(t)$ of the midpoint of the lower boundary is plotted in Figure 5 The truss is initially held in its deformed configuration for 5 seconds (shown in the first inset in Figure 5), before being released at the beginning of the dynamic simulation $(t=0 \mathrm{~s})$. The truss displays under-damped oscillations before returning to its undeformed configuration (shown in the last inset in Figure 5, with viscous damping being well captured in the simulation. The axial strains in the segments remain small as expected, given the high ratio of axial to bending stiffness. 


\subsection{Activation of natural curvature}

Shape memory polymers (SMPs) have the ability to change their shape when activated by an external stimulus such as heating, exposure to light, a chemical reaction, or a mechanical stimulus [45]. Structures made of SMPs can be designed to have several equilibrium shapes which are defined during the programming (or shape-fixing) process and typically include a temporary equilibrium shape and a permanent shape that is recovered upon activation. Remarkable examples are self-folding structures which consist of thin sheets with an as-designed natural curvature [46, 47, 48], used, e.g., to generate self-folding origami achieved through the careful design of fold locations and a control sequence [49]. Inspired by this concept, we show that our numerical framework can be used to predict the complex topology evolution of assemblies of beams or wires made of SMPs (also termed $4 D$ rods in [4]) used as flexural, torsional, and extensional actuators in the design of morphing structures [42, 43, 44].

A discrete beam with natural curvatures $\kappa_{0}^{1}, \kappa_{0}^{2}$ (along the material directors $\mathbf{d}_{1}$ and $\mathbf{d}_{2}$, respectively) is modeled [50] by modifying the bending contribution of the discrete elastic potential (13) according to

$$
\begin{aligned}
W_{i}\left(\mathbf{E}_{i}\right)= & \frac{\tilde{l}_{0}^{i}}{2}\left[E A \varepsilon_{i}^{2}+E I_{1}\left(\frac{\kappa_{i}^{1}}{\tilde{l}_{0}^{i}}-\kappa_{0}^{1}\right)^{2}+E I_{2}\left(\frac{\kappa_{i}^{2}}{\tilde{l}_{0}^{i}}-\kappa_{0}^{2}\right)^{2}\right] \\
& +\frac{\tilde{l}_{0}^{i}}{2}\left[\mu J\left(\frac{\tau_{i}}{\tilde{l}_{0}^{i}}\right)^{2}\right] .
\end{aligned}
$$

With this modified potential, we utilize the discrete beam and junction elements to simulate the experiments of [43], featuring a 2D truss that shrinks when the natural curvature in selected regions of the horizontal beams (viz., in those regions close to beam junctions) is activated, as shown in Figure 6 We define the natural curvature along the horizontal beams as $\left(\kappa_{1}^{0}, \kappa_{2}^{0}\right)=\left(0, \kappa_{0} / L\right)$ where $\mathbf{d}_{1}=\mathbf{e}_{y}, \mathbf{d}_{2}=\mathbf{e}_{z}$, $L$ denotes the horizontal beams' lengths, and we assign the final non-dimensional natural curvature $\kappa_{0}= \pm 14.31$. Starting with an undeformed configuration, we solve for quasistatic equilibrium in the presence of natural curvature. The thus obtained switchable patterns, illustrated in Figure 6a), can be used as a building block to produce various shapes upon tesselation, such as the example of the ETH logo from [43], here reproduced in Figure 6 b). In our simulations, we use homogeneous, isotropic, linear elastic beams with $I_{1}=I_{2}=$ $I, A L^{2} / I=10^{2}$, which are discretized into $10(60)$ segments per beam for the vertical (horizontal) struts.

In an extension of the above concept, we demonstrate how storing natural curvature in the out-of-plane direction of a planar square lattice can be used to achieve coordinated 3D actuation and to result in, e.g., in-plane shrinkage, reducing the structures footprint as shown in Figure 7. We here define the natural curvature as $\left(\kappa_{1}^{0}, \kappa_{2}^{0}\right)=\left(0, \kappa_{0} / L\right)$ where $L$ denotes the length of each beam.

The beams aligned with $\mathbf{e}_{x}$ are defined with $\mathbf{d}_{1}=\mathbf{e}_{z}$, $\mathbf{d}_{2}=-\mathbf{e}_{y}$, and $\mathbf{d}_{3}=\mathbf{t}=\mathbf{e}_{x}$, whereas all beams aligned with

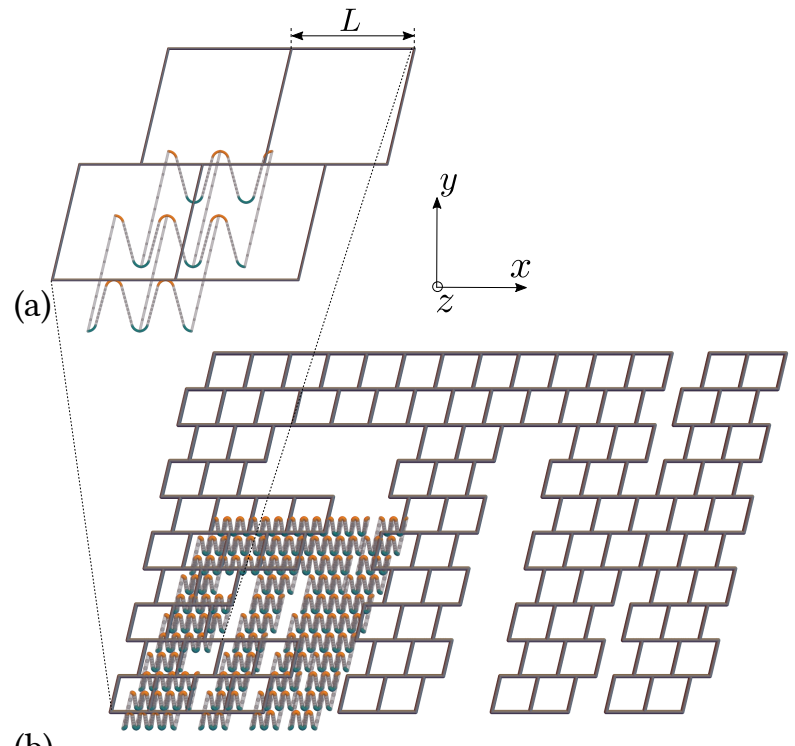

(b)

Fig. 6: Programmable elastomeric structure changing its shape upon thermal activation, which was demonstrated experimentally by [43]: the segments with positive nondimensional natural curvature $\kappa_{0}=14.31$ are highlighted in green (while segments with $\kappa_{0}=-14.31$ are colored in orange). Shown are the initial undeformed configuration (without natural curvature) and final equilibrium solution (with natural curvature) of (a) a single building block and (b) of a tesselation of building blocks into the ETH logo.

$\mathbf{e}_{y}$ are defined with $\mathbf{d}_{1}=\mathbf{e}_{z}, \mathbf{d}_{2}=\mathbf{e}_{x}$, and $\mathbf{d}_{3}=\mathbf{t}=\mathbf{e}_{y}$. We use the same constitutive behavior for all beams as in the previous example and conduct simulations with $30 \mathrm{seg}$ ments per beam. The predicted actuation mechanism, shown in Figure 7, illustrates the potential of out-of-plane natural curvature for applications from reconfigurable structures to robotics. Our discrete beam framework is hence beneficial to explore the design space by varying, e.g., the geometry, natural curvature, and orientation of the material frames.

\subsection{Cooperative buckling under axial expansion}

Unlike in the natural curvature-based examples above, shape-changing structures have also been reported in which cooperative buckling of (axially confined) beams is achieved by activating beams to elongate. For example, recent experiments by [12] exploited electrochemistry to induce a shape change in a square-shaped planar lattice whose Si-coated beams swell upon voltage-induced lithiation, leading to compressive axial eigenstrains. Due to pinned beam junctions (realized experimentally by stiff out-of-plane columns supporting each junction), the axial eigenstrains lead to cooperative buckling into a pattern reminiscent of that studied previously by [3].

We use our discrete beam framework to simulate the structural behavior of such a square lattice (lying in the $\mathbf{e}_{x^{-}}$ $\mathbf{e}_{y}$-plane) upon actuation. For this planar lattice we define the initial material frame orientation by $\mathbf{d}_{1}=\mathbf{e}_{z}$ for each 

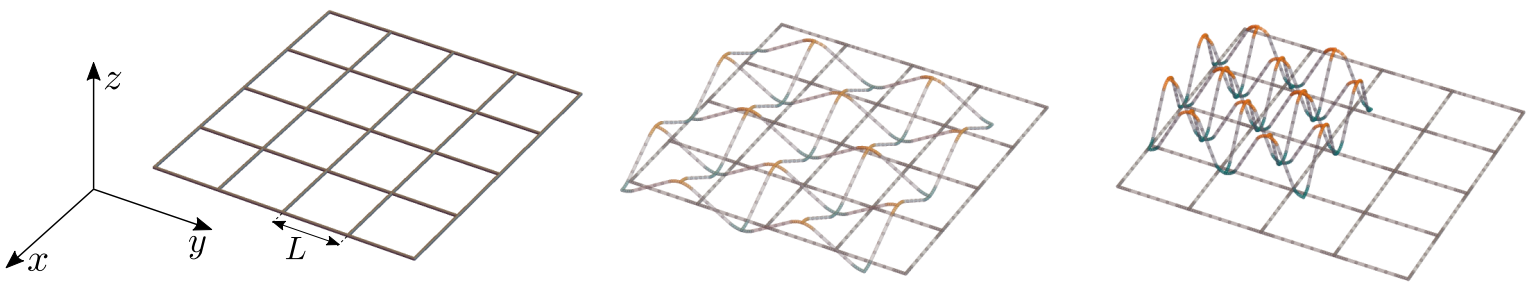

Fig. 7: Shape-changing programmable structure made of a square lattice with out-of-plane natural curvature $\kappa_{0}$ : the segments with positive (respectively, negative) natural curvature are highlighted in green (respectively, orange) with curvatures $\kappa_{0}=0$, $\kappa_{0}= \pm 2.815$, and $\kappa_{0}= \pm 5.745$ (from left to right).

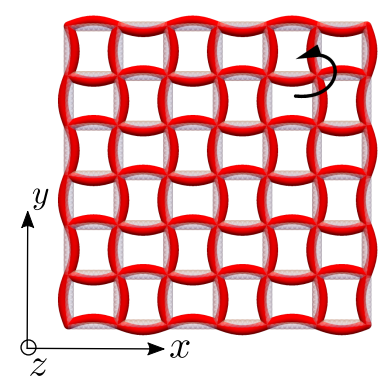

(a)

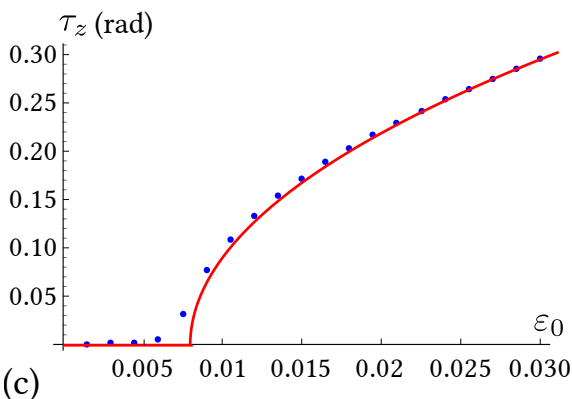

Fig. 8: Cooperative buckling under constraint axial expansion. A square truss lattice (whose junctions are pinned) buckles under a compressive axial pre-strain. tThe undeformed configuration (in gray) and deformed configuration for $\varepsilon_{0}=0.03$ (in red) are shown, obtained from simulations with two small initial imperfections and computed with 10 segments per beam. The orientation of the initial imperfections (small moments $M_{z}=0.1 E I / L$ ) are indicated by black arrows. In example (a) one axial moment is applied, in example (b) two axial moments of opposite senses are applied. (c) Results for the amplitude $\tau_{z}$ of the nodal rotations in a homogeneously buckled lattice as obtained from our framework (blue dots) are compared to the solution of the continuous equations for a single beam by continuation (red line) as a function of the eigenstrain $\varepsilon_{0}$.

beam in the lattice. To prevent out-of-plane buckling and torsion, we assume beams whose cross-section extends significantly more in the $\mathbf{e}_{z}$-direction, taking $I_{2}=J=10^{3} I_{1}$. As in experiments, the lattice junctions are pinned (to mimic the constraint by pillars on a fixed substrate), and the beams are weakly extensible with $A L^{2} / I_{1}=1.25 \cdot 10^{3}$. We impose an axial eigenstrain $\varepsilon_{0}$ by modifying the axial contribution to the discrete elastic potential (13), analogous to (16), resulting in

$$
\begin{aligned}
W_{i}\left(\mathbf{E}_{i}\right)= & \frac{\tilde{l}_{0}^{i}}{2}\left[E A\left(\varepsilon_{i}-\varepsilon_{0}\right)^{2}+E I_{1}\left(\frac{\kappa_{i}^{1}}{\tilde{l}_{0}^{i}}\right)^{2}+E I_{2}\left(\frac{\kappa_{i}^{2}}{\tilde{l}_{0}^{i}}\right)^{2}\right] \\
& +\frac{\tilde{l}_{0}^{i}}{2}\left[\mu J\left(\frac{\tau_{i}}{\tilde{l}_{0}^{i}}\right)^{2}\right] .
\end{aligned}
$$

To initiate buckling, we introduce a small perturbation moment $M_{z}$ at one of the lattice's junctions, as schematically shown in Figure $8(a)$. When the axial eigenstrain $\varepsilon_{0}$ increases, the beams cooperatively buckle, forming a regular pattern involving a rotation $\pm \tau_{z}$ of the welded joints, as shown in red in Figure $8\left(\right.$ a). We measure the amplitude $\tau_{z}$ of the rotation at nodes as a function of the eigenstrain $\varepsilon_{0}$, and we compare it with a prediction obtained by solving the continuous equations for a single pinned-pinned slender beam under axial expansion by continuation [51], showing good agreement in Figure 8(c). The difference with the continuous prediction near the bifurcation point is attributed to the small perturbation introduced in the simulations.

When more complex or spatially distributed initial imperfections are present, non-periodic buckling patterns can arise that involve homogeneous domains separated by domain walls [12]. This is demonstrated here within our simulation framework by applying an initial imperfection that is incompatible with the regular pattern. Specifically, we impose external moments of opposite senses at two selected junctions in the lattice, as schematically shown in Figure 8(b). Two distinct domains featuring opposite orientations of the pattern appear, separated by an interface (pictured as the shaded cells in Figure 8(b)), reproducing the experimental observation of domain formation in [12].

\subsection{Compressive buckling of anisotropic beams}

A novel manufacturing and deployment technique for complex 3D structures was proposed in recent years [8] 39], which exploits the self-assembly (i.e., the pop-up) of initially flat, multi-layered thin stuctures made of slender filaments, attached to a pre-strained substrate. By a careful selection of the in-plane geometry of the different layers, the position of 

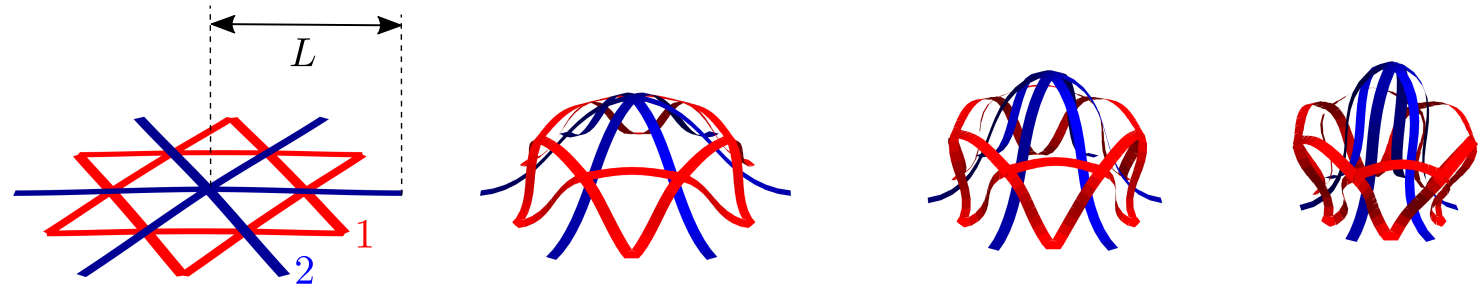

Fig. 9: Simulation of a pop-up structure demonstrated experimentally by [39]: an assembly of slender ribbons buckles under an equi-biaxial compression of $0 \%, 20 \%, 40 \%$ and $60 \%$ (respectively from left to right) induced through a prestretched substrate. Results were calculated using 10 discrete beam segments per each individual ribbon's segment. The two independent layers 1 and 2 are plotted in red and blue, respectively.

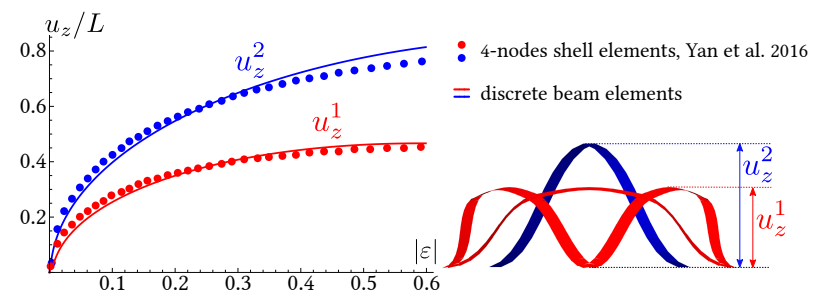

Fig. 10: Maximum rescaled out-of-plane displacement $u_{z} / L$ of each leayer's apex as a function of the applied equi-biaxial compressive strain magnitude $|\varepsilon|$ for the two layers pictured in Figure 9 . Compared are results from discrete beam simulations with 10 segments per beam (continuous curves in red and blue correspond to layers 1 and 2, respectively) and 2D shell elements from [39] (red and blue dots).

the bonding sites, and the level of pre-strain in the substrate, a variety of 3D shapes has been presented. Existing modeling work of the deformation of such slender filaments during assembly relies primarily on shell elements [40], which implies computationally expensive simulations compared to using a 1D beam formulation. Here, we show that our numerical framework is suitable for the analysis of such pop-up structures at a reduced computational cost.

As a representative example, we simulate the assembly process of the two-layer structure pictured in Figure 9 and originally described by [39]. The two independent layers (drawn in red and blue) consist of slender wires bonded at their free ends to a pre-stretched substrate. The identical cross-sections of all wires are thin (with a width-to-thickness aspect ratio of approximately 10), which is why we model them as inextensible Euler-Bernoulli beams with anisotropic cross-sections $\left(I_{2}=0.01 I_{1}, A=100 \pi I_{1} / L^{2}\right.$ and a torsion constant $\left.J=0.04 I_{1}\right)$. A progressive release of the pre-strain in the substrate yields a compressive equi-biaxial strain induced into the structures through the bonding sites, leading to outof-plane buckling of the two layers, as shown in Figure 9 In order to capture buckling in the simulations, we apply a set of small vertical forces of amplitude $f=10^{-2} E I_{1} / L^{2}$ at the center of the cross on the blue layer and at the junctions between the six branches of the star on the red layer. For a quantitative assessment of the simulation accuracy, we plot the maximal (normalized) out-of-plane displacement $u_{z} / L$ of each layer's apex (see Figure 10) as a function of the prestrain amplitude $|\varepsilon|$. Results of our discrete beam simulation are similar to those of [39] who used shell elements (of significantly higher resolution than the 1D elements employed here). Note that an in-depth quantitative comparison with a shell model falls into the scope of assessing the range of validity of beam theory when modeling ribbons, which is beyond the scope of the present work. Our aim is to show that the present approach is well suited for the study of such shape-morphing structures. Whether a model based on beams, shells, plates, ribbons, or a full 3D representation is sufficiently accurate and reasonably efficient, depends on the particular problem at hand (but is independent of the accuracy of the discrete beam formulation presented here). We note that in this specific example, a ribbon model [52,53] could possibly have achieved higher accuracy but would have required a specific re-implementation [21]. Since the aspect ratio of the cross-section is moderate, our simulation based on the discrete beam description provides a satisfactory and efficient alternative. In addition, naturally curved ribbons [41] could be described by extending our approach to a ribbon model such as the one proposed by [54].

\section{Conclusions}

We have presented a theoretical framework and its numerical realization for describing slender beam networks in a geometrically exact fashion, while accounting for large rotations. The setup is applicable to both elastic and inelastic as well as time-dependent base material constitutive laws. Based on the discrete beam description of [29], we here introduced a new approach to model rigid junctions between segments, and we extended the framework to account for anicotropic cross-sections (relevant for the modeling of ribbons), natural curvature and twist, as well as axial eigenstrains. We demonstrated the applicability of our discrete beam formulation to concepts of shape-morphing structures, including shape-changing elastomeric networks triggered by a change in natural curvature, the cooperative buckling of a truss lattices, and the self-assembly of 3D ribbon structures by compressive buckling. Of course, the model and code can be enriched in various directions, e.g., by integrating contact, friction, or more advanced 1D models for ribbons. As 
we believe that our numerical framework will be a useful tool for designers and researchers within the emergent field of $4 \mathrm{D}$ printing and active/adaptive structures to explore the available design space and actuation mechanisms, an opensource version of our code - used for all examples reported here - is available at github.com/lclaire/UtoBeams.

\section{Acknowledgements}

C.L. acknowledges the support from an ETH Postdoctoral Fellowship. We thank Manuel Weberndorfer for his contribution to the parallel implementation of the code and its integration with PETSc.

\section{References}

[1] Shankar, M. R., Smith, M. L., Tondiglia, V. P., Lee, K. M., McConney, M. E., Wang, D. H., Tan, L.-S., and White, T. J., 2013. "Contactless, photoinitiated snap-through in azobenzene-functionalized polymers". PNAS, 110, pp. 18792-18797.

[2] Gladman, A. S., Matsumoto, E. A., Nuzzo, R. G., Mahadevan, L., and Lewis, J. A., 2016. "Biomimetic 4D printing". Nature Materials, 15, pp. 413-419.

[3] Janbaz, S., Hedayati, R., and Zadpoor, A., 2016. "Programming the shape-shifting of flat soft matter: from self-rolling/self-twisting materials to self-folding origami". Materials Horizons, 3(6), pp. 536-547.

[4] Ding, Z., Weeger, O., Qi, H. J., and Dunn, M. L., 2018. "4D rods: 3D structures via programmable $1 \mathrm{~d}$ composite rods". Materials and Design, 137, p. 256.

[5] Lei, M., Hong, W., Zhao, Z., Hamel, C. M., Chen, M., Lu, H., and Qi, H. J., 2019. "3d printing of auxetic metamaterials with digitally reprogrammable shape". ACS applied materials \& interfaces.

[6] Testa, P., Style, R. W., Cui, J., Donnelly, C., Borisova, E., Derlet, P. M., Dufresne, E. R., and Heyderman, L. J., 2019. "Magnetically addressable shape-memory and stiffening in a composite elastomer". Advanced Materials, p. 1900561.

[7] Boley, J. W., van Rees, W. M., Lissandrello, C., Horenstein, M. N., Truby, R. L., Kotikian, A., Lewis, J. A., and Mahadevan, L., 2019. "Shape-shifting structured lattices via multimaterial 4d printing". Proceedings of the National Academy of Sciences, 116(42), pp. 2085620862.

[8] Xu, S., Yan, Z., Jang, K.-I., and Huang, W., 2015. "Assembly of micro/nanomaterials into complex, threedimensional architectures by compressive buckling". Science, 347, pp. 154-159.

[9] Duduta, M., Hajiesmaili, E., Zhao, H., Wood, R. J., and Clarke, D. R., 2019. "Realizing the potential of dielectric elastomer artificial muscles". Proceedings of the National Academy of Sciences, 116(7), pp. 2476-2481.

[10] Kotikian, A., McMahan, C., Davidson, E. C., Muhammad, J. M., Weeks, R. D., Daraio, C., and Lewis, J. A., 2019. "Untethered soft robotic matter with passive control of shape morphing and propulsion". Science Robotics, 4(33), pp. Art-No.

[11] Kim, Y., Parada, G. A., Liu, S., and Zhao, X., 2019. "Ferromagnetic soft continuum robots". Science Robotics, 4(33), p. eaax7329.

[12] Xia, X., Afshar, A., Yang, H., Portela, C. M., Kochmann, D. M., Di Leo, C. V., and Greer, J. R., 2019. "Electrochemically reconfigurable architected materials". Nature, 573(7773), pp. 205-213.

[13] Simo, J. C., 1985. "A finite strain beam formulation. The three-dimensional dynamic problem. Part I". Computer Methods in Applied Mechanics and Engineering, 49, pp. 55-70.

[14] Simo, J. C., and Vu-Quoc, L., 1986. "A threedimensional finite-strain rod model. Part II: Computational aspects". Computer Methods in Applied Mechanics and Engineering, 58, pp. 79-116.

[15] Cardona, A., and Geradin, M., 1988. "A beam finite element non-linear theory with finite rotations". International Journal for Numerical Methods in Engineering, 26, pp. 2403-2438.

[16] Ibrahimbegović, A., 1995. “On finite element implementation of geometrically nonlinear Reissner's beam theory: three-dimensional curved beam elements". Computer Methods in Applied Mechanics and Engineering, 122, pp. 11-26.

[17] Sonneville, V., Cardona, A., and Brüls, O., 2014. "Geometrically exact beam finite element formulated on the special euclidean group SE(3)". Computational Methods Appl. Mech. Engrg., 268, pp. 451-474.

[18] Bergou, M., Wardetzky, M., Robinson, S., Audoly, B., and Grinspun, E., 2008. "Discrete elastic rods". ACM transactions on graphics, 27, p. 63.

[19] Jung, P., Leyendecker, S., Linn, J., and Ortiz, M., 2011. "A discrete mechanics approach to the cosserat rod theory - part 1: static equilibria". International Journal for Numerical Methods in Engineering, 85, pp. 31-60.

[20] Jawed, M., Novelia, A., and OReilly, O., 2017. A Primer on the Kinematics of Discrete Elastic Rods. Springer.

[21] Shen, Z., Huang, J., Chen, W., and Bao, H., 2015. "Geometrically exact simulation of inextensible ribbon”. Computer Graphics Forum, 34, pp. 145-154.

[22] Bergou, M., Audoly, B., Vouga, E., Wardetzky, M., and Grinspun, E., 2010. "Discrete viscous threads". ACM Transactions on Graphics, 29.

[23] Audoly, B., Clauvelin, N., Brun, P.-T., Bergou, M., Grinspun, E., and Wardetzky, M., 2013. "A discrete geometric approach for simulating the dynamics of thin viscous threads". Journal of Computational Physics, 253, pp. 18-49.

[24] Lang, H., Linn, J., and Arnold, M., 2011. "Multi-body dynamics simulation of geometrically exact cosserat rods". Multibody System Dynamics, 25(3), pp. 285312.

[25] Linn, J., Lang, H., and Tuganov, A., 2013. "Geometrically exact cosserat rods with Kelvin-Voigt type viscous damping”. Mechanical Sciences, 4(1), pp. 79-96. 
[26] Jawed, M. K., Da, F., Joo, J., Grinspun, E., and Reis, P., 2014. "Coiling of elastic rods on rigid substrates". PNAS, 111, pp. 14663-14668.

[27] Kaufman, D. M., Tamstorf, R., Smith, B., Aubry, J.M., and Grinspun, E., 2014. "Adaptive nonlinearity for collisions in complex rod assemblies". ACM Trans. on Graphics (SIGGRAPH 2014).

[28] Gazzola, M., Dudte, L. H., McCormick, A. G., and Mahadevan, L., 2018. "Forward and inverse problems in the mechanics of soft filaments". Royal Society Open Science, $\mathbf{5}$.

[29] Lestringant, C., Audoly, B., and Kochmann, D. M., 2020. "A discrete, geometrically exact method for simulating nonlinear, elastic and inelastic beams". Computer Methods in Applied Mechanics and Engineering, 361, p. 112741.

[30] Ortiz, M., and Stainier, L., 1999. "The variational formulation of viscoplastic constitutive updates". Computer Methods in Applied Mechanics and Engineering, 171, pp. 419-444.

[31] Crisfield, M. A., 1990. "A consistent co-rotational formulation for non-linear, three-dimensional, beamelements". Computer Methods in Applied Mechanics and Engineering, 81(2), pp. $131-150$.

[32] Crisfield, M. A., and Jelenić, G., 1998. "Objectivity of strain measures in the geometrically exact threedimensional beam theory and its finite-element implementation". Proc. R. Soc. A, 455, p. 11251147.

[33] Perez, J., Thomaszewski, B., Coros, S., Bickel, B., Canabal, J. A., Sumner, R., and Otaduy, M. A., 2015. "Design and fabrication of flexible rod meshes". ACM Transactions on Graphics, 34, p. 138.

[34] Dalcin, L., Kler, P., Paz, R., and Cosim, A., 2011. "Parallel distributed computing using Python". Advances in Water Resources, 34(9), pp. 1124-1139.

[35] Phlipot, G. P., and Kochmann, D. M., 2019. "A quasicontinuum theory for the nonlinear mechanical response of general periodic truss lattices". Journal of the Mechanics and Physics of Solids, 124, pp. 758-780.

[36] Wicks, N., and Guest, S. D., 2004. "Single member actuation in large repetitive truss structures". International Journal of Solids and Structures, 41(3-4), pp. 965-978.

[37] Leung, A., and Guest, S., 2007. "Single member actuation of kagome lattice structures". Journal of Mechanics of Materials and Structures, 2(2), pp. 303-317.

[38] Paulose, J., Meeussen, A. S., and Vitelli, V., 2015. "Selective buckling via states of self-stress in topological metamaterials". Proceedings of the National Academy of Sciences, 112(25), pp. 7639-7644.

[39] Yan, Z., Zhang, F., Liu, F., Han, M., Ou, D., Liu, Y., Lin, Q., Guo, X., Fu, H., Xie, Z., et al., 2016. "Mechanical assembly of complex, $3 \mathrm{~d}$ mesostructures from releasable multilayers of advanced materials". Science advances, 2(9), p. e1601014.

[40] Yan, Z., Han, M., Yang, Y., Nan, K., Luan, H., Luo, Y., Zhang, Y., Huang, Y., and Rogers, J. A., 2017. "Deterministic assembly of $3 \mathrm{~d}$ mesostructures in advanced materials via compressive buckling: A short review of recent progress". Extreme Mechanics Letters, 11, pp. 96-104.

[41] Guo, X., Wang, X., Ou, D., Ye, J., Pang, W., Huang, Y., Rogers, J. A., and Zhang, Y., 2018. "Controlled mechanical assembly of complex $3 \mathrm{~d}$ mesostructures and strain sensors by tensile buckling". npj Flexible Electronics, 2(1), p. 14.

[42] Raviv, D., Zhao, W., McKnelly, C., Papadopoulou, A., Kadambi, A., Shi, B., Hirsch, S., Dikovsky, D., Zyracki, M., Olguin, C., Raskar, R., and Tibbits, S., 2014. "Active printed materials for complex selfevolving deformations". Scientific Reports, 4.

[43] Wagner, M., Chen, T., and Shea, K., 2017. "Large shape transforming 4D auxetic structures". 3D Printing and Additive Manufacturing, 4, pp. 133-141.

[44] Ding, Z., Yuan, C., Peng, X., Wang, T., Qi, H. J., and Dunn, M. L., 2017. "Direct 4d printing via active composite materials". Science Advances, 3.

[45] Zhao, Q., Qi, H. J., and Xie, T., 2015. "Recent progress in shape memory polymer: New behavior, enabling materials, and mechanistic understanding". Progress in Polymer Science, 49-50, pp. 79-120.

[46] Mailen, R. W., Liu, Y., Dickey, D., Zikry, M., and Genzer, J., 2015. "Modelling of shape memory polymer sheets that self-fold in response to localized heating". Soft Matter, 11, p. 7827.

[47] Mao, Y., Isakov, M. S., Wu, J., Dunn, M. L., and Qi, H. J., 2015. "Sequential self-folding structures by $3 \mathrm{~d}$ printed digital shape memory polymers". Scientific Reports, 5.

[48] Hubbard, A. M., Mailen, R. W., Zikry, M., Dickey, D., and Genzer, J., 2017. "Controllable curvature from planar polymer sheets in response to light". Soft Matter, 13, p. 2299.

[49] Hawkes, E., An, B., Bendernou, N. M., Tanaka, H., Kim, S., Demaine, E. D., Rus, D., and Wood, R. J., 2010. "Programmable matter by folding". Proc. Natl. Acad. Sci. U.S.A., 107, p. 12441.

[50] Basset, A. B., 1895. "On the deformation of thin elastic wires.". American Journal of Mathematics, 17, pp. 281-317.

[51] Doedel, E. J., Champneys, A. R., Fairgrieve, T. F., Kuznetsov, Y. A., Sandstede, B., and Wang, X. J., 2007. AUTO-07p: continuation and bifurcation software for ordinary differential equations. See http://indy.cs.concordia.ca/auto/.

[52] Sadowsky, M., $1930 . \quad$ "Theorie der elastisch biegsamen undehnbaren bänder mit anwendungen auf das möbiussche band. verh. 3. intern. kongr. techn". Mechanik (Stockholm 1930), II, pp. 444-451.

[53] Wunderlich, W., 1962. "Über ein abwickelbares möbiusband". Monatshefte für Mathematik, 66(3), pp. 276-289.

[54] Audoly, B., and Seffen, K. A., 2016. "Buckling of naturally curved elastic strips: the ribbon model makes a difference". In The Mechanics of Ribbons and Möbius Bands. Springer, pp. 293-320. 


\section{A Parametrization of rigid body rotations}

As discussed in Section 2.2, the strain measures required in a junction element are expressed by means of the rigidbody rotation at the junction, which is parametrized by a quaternion $\mathbf{q}$. With this parametrization, we need to compute the variations of the associated rotation tensor $\mathbf{R}(\mathbf{q})$ to calculate internal forces and the consistent stiffness matrix of the junction element (the latter is required for implicit solvers, such as the Newton-Raphson solver used for all quasistatic boundary value problems in this study).

The quaternion $\mathbf{q}$ parametrizing the rotation writes

$$
\mathbf{q}=\bar{a}+\bar{b} \mathbf{i}+\bar{c} \mathbf{j}+\bar{d} \mathbf{k}
$$

with $(\bar{a}, \bar{b}, \bar{c}, \bar{d}) \in \mathbb{R}^{4}$, and $\mathbf{i}, \mathbf{j}, \mathbf{k}$ being the fundamental quaternion units. The associated unit quaternion is defined as

$$
\overline{\mathbf{q}}(\mathbf{q})=\frac{\mathbf{q}}{|\mathbf{q}|}=a+b \mathbf{i}+c \mathbf{j}+d \mathbf{k},
$$

where $|\mathbf{q}|=\bar{a}^{2}+\bar{b}^{2}+\bar{c}^{2}+\bar{d}^{2}$. The components of the corresponding rotation tensor in the Cartesian reference frame are obtained as

$$
\begin{aligned}
& \mathbf{R}(\overline{\mathbf{q}})= \\
& {\left[\begin{array}{ccc}
a^{2}+b^{2}-c^{2}-d^{2} & 2 b c-2 a d & 2 a c+2 b d \\
2 b c+2 a d & a^{2}-b^{2}+c^{2}-d^{2} & 2 c d-2 a b \\
-2 a c+2 b d & 2 c d+2 a b & a^{2}-b^{2}-c^{2}+d^{2}
\end{array}\right] .}
\end{aligned}
$$

Note that, when the rotation is the identity, the corresponding quaternion is given by $(a, b, c, d)=(1,0,0,0)$.

In order to derive the residual force vector and stiffness matrix used in the numerical scheme, we need explicit expressions for the first and second variations of the various geometric quantities involved in the calculation of the strains. Variations of segments, unit tangent vectors, holonomy, material frame vectors and the binormal vector were provided in [29]. Here, we complement the description by calculating the first and second variations of the rotation $\mathbf{R}$ for infinitesimal perturbations of the degrees of freedom stored in the quaternion $\mathbf{q}$, defined by (19) and (20). Since $\mathbf{R}$ depends on the unit quaternion $\overline{\mathbf{q}}(\mathbf{q})$ through $(20)$, the variations of $\mathbf{R}$ involve the variations of $\overline{\mathbf{q}}$. For an infinitesimal perturbation of $\mathbf{q}$, denoted $\delta \mathbf{q}$, the first variation writes

$$
\delta \overline{\mathbf{q}}=\frac{\mathbf{I}_{4}-\mathbf{q} \otimes \mathbf{q}}{|\mathbf{q}|} \cdot \delta \mathbf{q}
$$

where $\mathbf{I}_{4}$ is the identity on vectors in $\mathbb{R}^{4}$. The second variation for a unit quaternion can be expressed in a similar way, given two infinitesimal perturbations of $\mathbf{q}$, denoted $\delta \mathbf{q}_{1}$ and $\delta \mathbf{q}_{2}$, as

$$
\begin{aligned}
\delta^{2} \overline{\mathbf{q}}= & -\frac{\left(\mathbf{q} \cdot \delta \mathbf{q}_{1}\right) \delta \mathbf{q}_{2}+\left(\mathbf{q} \cdot \delta \mathbf{q}_{2}\right) \delta \mathbf{q}_{1}+\left(\delta \mathbf{q}_{2} \cdot \delta \mathbf{q}_{1}\right) \mathbf{q}}{|\mathbf{q}|^{3}} \\
& +3 \frac{\left(\mathbf{q} \cdot \delta \mathbf{q}_{1}\right)\left(\mathbf{q} \cdot \delta \mathbf{q}_{2}\right) \mathbf{q}}{|\mathbf{q}|^{5}}
\end{aligned}
$$

Finally, the first and second variations of the rotation tensor write, respectively,

$$
\delta \mathbf{R}=\frac{\partial \mathbf{R}}{\partial \overline{\mathbf{q}}} \cdot \delta \overline{\mathbf{q}} \quad \text { and } \quad \delta^{2} \mathbf{R}=\frac{\partial^{2} \mathbf{R}}{\partial \overline{\mathbf{q}}^{2}} \cdot \delta \overline{\mathbf{q}}+\frac{\partial \mathbf{R}}{\partial \overline{\mathbf{q}}} \cdot \delta^{2} \overline{\mathbf{q}}
$$

where the components of $\partial \mathbf{R} / \partial \overline{\mathbf{q}}$ and $\partial^{2} \mathbf{R} / \partial \overline{\mathbf{q}}^{2}$ are found by differentiating 20) with respect to the components of $\overline{\mathbf{q}}$. Finally, we introduce a basis for quaternions as $\left(\hat{\mathbf{q}}_{k}\right)_{k \in\{1,2,3,4\}} \in$ $\mathbb{R}^{4}$ defined as

$$
\begin{aligned}
& \hat{\mathbf{q}}_{1}=(1,0,0,0), \hat{\mathbf{q}}_{2}=(0,1,0,0), \\
& \hat{\mathbf{q}}_{3}=(0,0,1,0), \hat{\mathbf{q}}_{4}=(0,0,0,1),
\end{aligned}
$$

which will be used for projection in the following two sections.

\section{B Spin angle at a ghost segment}

The spin angle at a ghost segment is implicitly defined by Eq. (6). Here, we provide an explicit expression for this angle and for its variations (required for the calculation of internal forces and consistent tangent matrices of a junction element), assuming that the rotation at a ghost segment, parametrized by $\mathbf{q}^{i}$, is finite (i.e., when the current configuration is far from the reference configuration); the other case is discussed in Section 2.2

If $\tilde{\mathbf{t}}^{i} \neq \tilde{\mathbf{t}}_{\star}^{i}$, we define the binormal unit vector

$$
\mathbf{b}^{i}=\frac{\tilde{\mathbf{t}}_{\star}^{i} \times \tilde{\mathbf{t}}^{i}}{\left|\tilde{\mathbf{t}}_{\star}^{i} \times \tilde{\mathbf{t}}^{i}\right|}
$$

Note that $\mathbf{R}^{i} \cdot \mathbf{b}^{i}$ is in the plane spanned by $\mathbf{b}^{i}$ and $\tilde{\mathbf{t}}^{i} \times \mathbf{b}^{i}$, and its coordinates in this basis are

$$
\mathbf{b}^{i} \cdot \mathbf{R}^{i} \cdot \mathbf{b}^{i}=\cos \tilde{\omega}^{i} \quad \text { and } \quad\left(\tilde{\mathbf{t}}^{i} \times \mathbf{b}^{i}\right) \cdot \mathbf{R}^{i} \cdot \mathbf{b}^{i}=\sin \tilde{\omega}^{i}
$$

We define the two scalar-valued functions $f_{i}$ and $g_{i}$ as $f_{i}\left(\mathbf{q}^{i}\right)=\mathbf{b}^{i} \cdot \mathbf{R}^{i} \cdot \mathbf{b}^{i}$ and $g_{i}\left(\mathbf{q}^{i}\right)=\left(\tilde{\mathbf{t}}^{i} \times \mathbf{b}^{i}\right) \cdot \mathbf{R}^{i} \cdot \mathbf{b}^{i}$ such that the spin angle $\tilde{\omega}^{i}$ is calculated using the 2-argument arc-tangent function with $\cos \tilde{\omega}^{i}=f_{i}\left(\mathbf{q}^{i}\right)$ and $\sin \tilde{\omega}^{i}=g_{i}\left(\mathbf{q}^{i}\right)$.

A special case occurs when the rotation parametrized by $\mathbf{q}^{i}$ is finite and $\tilde{\mathbf{t}}^{i}=\tilde{\mathbf{t}}_{\star}^{i}$. This implies that $\mathbf{q}^{i}$ represents a rotation about $\tilde{\mathbf{t}}_{\star}^{i}$ and the parallel transport in $(6)$ equals the identity, i.e., $\mathbf{R}\left(\tilde{\mathbf{t}}_{\star}^{i}, \tilde{\boldsymbol{\omega}}^{i}\right)=\mathbf{R}^{i}$. Further, writing $\overline{\mathbf{q}}^{i}=a+b \mathbf{i}+$ 
$c \mathbf{j}+d \mathbf{k}$ for the unit quaternion associated with $\mathbf{q}^{i}$, according to (19), the rotation angle $\widetilde{\omega}^{i}$ is characterized by

$$
\cos \tilde{\omega}^{i}=a \quad \text { and } \quad \sin \tilde{\omega}^{i}=\sqrt{b^{2}+c^{2}+d^{2}} .
$$

In this case we define the two scalar-valued functions $\tilde{f}_{i}\left(\mathbf{q}^{i}\right)=\overline{\mathbf{q}}^{i} \cdot \hat{\mathbf{q}}_{1}$ and $\tilde{g}_{i}\left(\mathbf{q}^{i}\right)=\sqrt{\sum_{l=2}^{4}\left(\overline{\mathbf{q}}^{i} \cdot \hat{\mathbf{q}}_{l}\right)^{2}}$, using the basis for quaternions introduced in (24), and we calculate the spin angle $\tilde{\omega}^{i}$ using the 2-argument arc-tangent function with $\cos \tilde{\omega}^{i}=\tilde{f}_{i}\left(\mathbf{q}^{i}\right)$ and $\sin \tilde{\omega}^{i}=\tilde{g}_{i}\left(\mathbf{q}^{i}\right)$.

Again, we present variations to complete the calculation of internal forces and consistent tangents. The case when the current configuration is close to the reference configurations is straightforward; differentiating (8) yields

$$
\delta \tilde{\omega}^{i}=\frac{1}{2}\left[\left(\mathrm{D} \mathbf{P}_{\tilde{\mathfrak{t}}^{i}}^{\tilde{f}_{\star}^{i}} \cdot \delta \mathbf{q}\right) \cdot \mathbf{R}^{i}+\mathbf{P}_{\tilde{\mathfrak{t}}^{i}}^{\tilde{\mathfrak{t}}^{i}} \cdot\left(\mathrm{D} \mathbf{R}^{i} \cdot \delta \mathbf{q}\right)\right]:\left(\tilde{\mathbf{t}}_{\star}^{i}\right)_{\times}
$$

An expression for $\delta \mathbf{R}^{i}=\mathrm{D} \mathbf{R}^{i} \cdot \delta \mathbf{q}$ is found by differentiating (20), and the variation of the parallel transport relation writes [29]

$$
\mathrm{D} \mathbf{P}_{\tilde{\mathbf{t}}^{i}}^{\tilde{\tilde{t}}^{i}} \cdot \delta \mathbf{q}=\left(\left(\delta \mathbf{R}^{i} \cdot \tilde{\mathbf{t}}_{\star}^{i}\right) \cdot \tilde{\mathbf{t}}_{\star}^{i}\right) \mathbf{1}+\left(\left(\delta \mathbf{R}^{i} \cdot \tilde{\mathbf{t}}_{\star}^{i}\right) \times \tilde{\mathbf{t}}_{\star}^{i}\right)_{\times} .
$$

When the rotation $\mathbf{R}^{i}$ is finite, the first variation $\delta \tilde{\omega}^{i}$ is found, using (26), as

$$
\delta \tilde{\omega}^{i}=-\left(\mathrm{D} f_{i}\left(\mathbf{q}^{i}\right) \cdot \delta \mathbf{q}\right) g_{i}\left(\mathbf{q}^{i}\right)+\left(\mathrm{D} g_{i}\left(\mathbf{q}^{i}\right) \cdot \delta \mathbf{q}\right) f_{i}\left(\mathbf{q}^{i}\right),
$$

or, using [27], as

$$
\delta \tilde{\omega}^{i}=-\left(\mathrm{D} \tilde{f}_{i}\left(\mathbf{q}^{i}\right) \cdot \delta \mathbf{q}\right) \tilde{g}_{i}\left(\mathbf{q}^{i}\right)+\left(\mathrm{D} \tilde{g}_{i}\left(\mathbf{q}^{i}\right) \cdot \delta \mathbf{q}\right) \tilde{f}_{i}\left(\mathbf{q}^{i}\right) .
$$

\section{Applying and measuring a moment at a junction}

Since rotations are not explicit degrees of freedom at vertices as, e.g., in corotational beam elements, we here derive an explicit expression for the generalized forces associated with a moment $M$ about an axis $\mathbf{u}$, measured or applied at a beam junction whose rotation is parametrized by $\mathbf{q}$. Let us define the unit vectors $\mathbf{v}_{1}^{\perp}$ and $\mathbf{v}_{2}^{\perp}$ such that $\left(\mathbf{u}, \mathbf{v}_{1}^{\perp}, \mathbf{v}_{2}^{\perp}\right)$ is an orthonormal basis. The components of the generalized nodal force vector $\mathbf{F}$ at the junction are related to $M$ by

$$
F_{k}=M \mathrm{D} \theta_{\mathbf{u}} \cdot \hat{\mathbf{q}}_{k},
$$

where $\left(\hat{\mathbf{q}}_{k}\right)_{k \in\{1,2,3,4\}}$ is the basis for quaternions introduced in 24, and $\theta_{\mathbf{u}}\left(\mathbf{q}^{i}\right)$ is characterized by

$$
\cos \theta_{\mathbf{u}}=\left(\mathbf{R}(\mathbf{q}) \cdot \mathbf{v}_{1}^{\perp}\right) \cdot \mathbf{v}_{1}^{\perp}, \quad \sin \theta_{\mathbf{u}}=\left(\mathbf{R}(\mathbf{q}) \cdot \mathbf{v}_{1}^{\perp}\right) \cdot \mathbf{v}_{2}^{\perp}
$$

